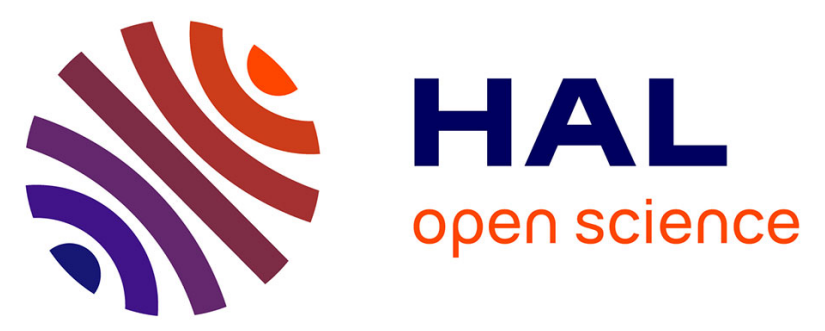

\title{
Hybrid Reasoning-Based Medical Platform to Assist Clinicians in their Clinical Reasoning Process
}

Lamine Benmimoune, Amir Hajjam, Parisa Ghodous, Emmanuel Andres, Samy Talha, Mohamed Hajjam

\section{To cite this version:}

Lamine Benmimoune, Amir Hajjam, Parisa Ghodous, Emmanuel Andres, Samy Talha, et al.. Hybrid Reasoning-Based Medical Platform to Assist Clinicians in their Clinical Reasoning Process. Information, Intelligence, Systems and Applications (IISA), 2015 6th International Conference, Jul 2015, Corfu, Greece. pp.1-5. hal-01263364

\section{HAL Id: hal-01263364 \\ https://hal.science/hal-01263364}

Submitted on 27 Jan 2016

HAL is a multi-disciplinary open access archive for the deposit and dissemination of scientific research documents, whether they are published or not. The documents may come from teaching and research institutions in France or abroad, or from public or private research centers.
L'archive ouverte pluridisciplinaire HAL, est destinée au dépôt et à la diffusion de documents scientifiques de niveau recherche, publiés ou non, émanant des établissements d'enseignement et de recherche français ou étrangers, des laboratoires publics ou privés. 


\title{
Hybrid Reasoning-Based Medical Platform to Assist Clinicians in their Clinical Reasoning Process
}

\author{
Lamine Benmimoune*†, Amir Hajjam*, Parisa Ghodous ${ }^{\ddagger}$, Emmanuel Andres ${ }^{\S}$, Samy Talha ${ }^{\S}$, Mohamed Hajjam ${ }^{\dagger}$ \\ *IRTES-SET, Université de Technologie Belfort-Montbéliard, 90010, Belfort, France \\ $\dagger$ Newel, 68100, Mulhouse, France \\ ‡LIRIS, Université Claude Bernard Lyon 1, 69622, Villeurbanne, France \\ ${ }^{\S}$ Hôpital Civil de Strasbourg, 67000 Strasbourg, France \\ Email: \{lamine.benmimoune, amir.hajjam-el-hassani\}@utbm.fr
}

\begin{abstract}
Clinical decision support systems (CDSSs) have been hailed for their potential to reduce medical errors and improve the quality and safety of health care. CDSSs aim to assist clinicians in their decision making and take over some routines including diagnostic support. This paper presents a design of a generic medical platform to assist clinicians in their clinical reasoning process. Our platform aims to guide clinicians in the process of gathering relevant information and to help them in their decision making. It exploits two sources of knowledge namely: theoretical knowledge (clinical rules) and practical knowledge (clinical experiences) by relying on RuleBased Reasoning and Case-Based Reasoning, respectively. moreover, we utilize the semantic web and ontologies for knowledge modeling in order to reuse and to share knowledge.
\end{abstract}

Keywords-Clinical Decision Support System, Ontology, Information Gathering System, Rule-Based Reasoning, Case-Based Reasoning, Clinical Reasoning, Clinical guidelines.

\section{INTRODUCTION}

Clinical reasoning cycle involves diverse cognitive activities, including gathering information, processing them, coming to an understanding of a patient problem, planning and implementing interventions, evaluating outcomes, reflecting on and learning from the process [1], [2], [3]

In the clinical reasoning cycle, gathering relevant information (cues) from the patient is crucial as it permits to guide the clinician in his reflection by exploiting different assumptions based on his/her theoretical knowledge and practical knowledge (experience).

In addition, the decision-making depends on the gathered information, to the point where any missing important information might lead the clinician to an error.

For these reasons, the gathering information process should be oriented, precise, detailed as it is useful in formulating a diagnosis and providing medical care to the patient [4].

In this paper, we present a novel approach to design an medical platform to assist clinicians in their clinical reasoning process including information gathering and decision making. Our platform permits more interaction with clinicians by guiding them in their clinical reflection. The main idea consists of generating dynamically personalized questions in connection with the patient's medical background. Each question is relevant and permits to confirm or to reject the assumptions formulated by the clinician. The questions are adapted to the patient profile, and take into account the clinical rules and the similar clinical cases already treated in the past as practiced by experimented clinicians.

In the proposed approach we rely on the use of two modes of reasoning namely: the Rule-Based Reasoning (RBR) and the Case-Based Reasoning (CBR) which permit to exploit theoretical knowledge (i.e. clinical rules) and practical knowledge (i.e clinical experiences), respectively.

The remainder of this paper is structured as follows. In the next section we present related work on clinical decision support system. In Section III we give a overview of different components of the proposed platform and we illustrate its general functioning. Finally, Section IV summarizes our contribution and concludes the paper.

\section{RELATED WORK}

Clinical Decision Support Systems (CDSSs) are tools which are designed to assist clinicians for better clinical decision-making with knowledge and relevant clinical data of the patient, intelligently filtered and presented, to enhance health and health care [5], [6], [7].

These tools are used in a variety of medical application such as diagnosis support, providing personalized protocols, computerized alerts and reminders to care providers, etc.

In the literature many studies have been conducted to categorize the CDSS, among which [6] and [8] have proposed to categorize CDSS into two mainly categories: Non-knowledgebased CDSSs and Knowledge-based CDSSs.

(i) Non-knowledge-based CDSSs: employ machine learning and data mining approaches. They generally use statistical learning algorithms such as neural networks, genetic algorithms, etc. Where machines learn from examples in order to discover patterns and visible or hidden relationships between clinical data [8].

(ii) Knowledge-based CDSSs: use experts' knowledge relating a specific domain and try to resolve problems by imitating the clinician reasoning. This knowledge is stored in a knowledge base in a standard and exploitable format [9]. The knowledge based CDSSs can use several reasoning mechanism such as Rule-Based Reasoning and Case-Based Reasoning. 


\section{A. Rule-Based CDSSs}

Rule-Based CDSSs are Knowledge-Based systems which use expert knowledge. The knowledge most often take the form of IF-THEN rules [8]. These rules are usually obtained from medical clinical guidelines that are elaborated by domain experts (clinicians).

In medical field several Rule-Based CDSSs are proposed in this area.

Goldstein et al. [10] and Lai et al. [11] present ATHENA a Rule-Based CDSS that provides recommendations for managing hypertension in primary care using the EON architecture. The rules are derived from medical clinical guidelines that are modeled by ontologies.

Rector and Rogers [22] propose a Rule-Based CDSS for preoperative risk assessment. The proposed CDSS is based on the use of ontologies for adapted information collection and patient representation. First, it gathers patient medical history through questionnaire ontology and then, the rules engine processes the collected information to infer patient risk assessment prior to surgery.

Sherimon et al. [24], [25] propose a Rule-Based CDSS for predicting the risk of hypertension and diabetes in related diseases. The proposed CDSS is based on the use of ontologies and clinical guidelines which provide rules to the inference engine.

Farooq et al. [26] propose an Ontology-Based approach to design a Rule-Based CDSS for chest pain risk assessment. The patient data (cardiovascular history and family History) is collected through a questionnaire-ontology as per the recommendations of $\mathrm{NICE}^{1}$ guidelines.

\section{B. Case-Based CDSSs}

Case-Based CDSSs are Knowledge-Based systems which are designed to work in the same way with the basic idea of similar problems have similar solutions. For solving a new problem, the system applies previous experiences adapted to the current situation [23], [28].

A Case-Based CDSS is mainly composed of two parts: a case base and a reasoning engine. The case base, contains all resolved cases in the past, and the reasoning engine resolves new problems based on the solutions of similar past cases.

The case refers to the clinician's experience in the solving of a specific problem[12]. Usually the case is composed of two parts (problem description and solution), but it can be extended by a third part (outcome) [19] [23].

(i) Problem description: describes specific conditions, states of a situation, when this particular case is recorded. For medical applications a problem description could describe a medical condition of the patient (signs, symptoms, laboratory tests, etc.).

(ii) Solution: provides how the problem described in the problem description was solved or treated in a particular instance. The solution of the problem could be diagnostic, treatment, etc.

\footnotetext{
${ }^{1}$ National Institute of Clinical Excellence
}

(iii) Outcome: describes the final result or consequence and feedback gained from the proposed solution. The outcome could be the medical condition of the patient after treatment.

In medical field several Case-Based CDSSs are proposed in this area.

El Balaa et al. [15], [16] propose FM-Ultranet that is a Case-Based CDSS. This system aims to help ultrasonographists to improve the early detection of fetal malformations through ultrasonographical examinations. The case base consists of 130 cases which are arranged in a hierarchical object structure. Data collection is done through a single form that offer all entries for a sane fetus.

Chakraborty et al. [17] propose SFDA which is a CaseBased CDSS, developed to assist diagnosis of Swine Flu. The case base consists of several medical cases including the common symptoms one encounters in patients suffering from Swine Flu and their corresponding prescriptions. The clinical data is entered through a form, and after the system return back the list of the most matched cases.

Begum [19] proposes a Hybrid CDSS to establish diagnosis and treatment plan in the psycho-physiological domain. The proposed CDSS is based on the Case-Based Reasoning and fuzzy techniques to handle uncertainty existent in clinician's reasoning process.

Wang and $\mathrm{A}$. Uz [20] propose a design of a template case store, called MedCase, which utilizes the semantic web technologies (ontologies) intended for case-based CDSS with distributed architecture.

The previous studies have allowed the designing of CDSSs to help clinicians in their clinical tasks in several medical domains based either on the RBR methodology or on the CBR methodology.

Although, the RBR methodology and the CBR methodology present many advantages by offering an easy representation of knowledge and learning capacity, respectively, the use of the RBR and the CBR might present some disadvantages. As reported by Begum [19] to apply a RBR, the domain should be well understood, and should remain constant over time which is not the case with the medical domain, since this presents a lot of complexity and is constantly evolving.

Furthermore, among the disadvantages of using CBR is that the structure of the cases are generally not designed to evolve [21]. In addition, a Case-Based CDSS is less efficient at the first use, when the case base is empty or it doesn't contain a lot of cases. Which means that its efficiency is dependent on the cases stored in the case base.

To palliate the disadvantages cited, we have proposed an hybrid methodology (see section III) which is based on the use of the case-based reasoning and the rule-based reasoning, as we consider that these two modes of reasoning can complement each other.

\section{PROPOSED APPROACH}

In this section we present our approach which consists of designing an hybrid medical platform to assist clinicians in their clinical reasoning process. The proposed platform is 
based on the use of ontologies and combines the two modes of reasoning $\mathrm{RBR}$ and $\mathrm{CBR}$. It aims to guide the clinicians in gathering relevant information about the patient's health status and to help them in their clinical decision making.

The RBR and CBR permit to exploit theoretical sources (Clinical Rules) and practical sources (Clinical Cases), respectively. The rules are defined by domain experts using medical guidelines which are provided by accredited organizations such as $\mathrm{AHA}^{2}$ and $\mathrm{ESC}^{3}$ for cardiovascular diseases.

The cases permit to capture the clinician's past experiences in the aim of reusing them to treat new clinical cases. The use of the CBR and the RBR in the system permits to take full advantage of each of the them and to ensure more accurate decision making.

In addition, the proposed platform uses Ontologies for representing data and domain knowledge as they offer many advantages: (i) Sharing the knowledge in the same domain; (ii) Reusing of the already build ontologies and (iii) Separating domain knowledge from operation knowledge [27]. Moreover, the use of the ontologies allows the use of semantic-web rule engine in the RBR, facilitate the cases retrieval and case matching purpose in the CBR [20].

\section{A. Architecture}

Fig. 1 gives an overview of the architecture of the proposed platform. The proposed architecture is composed of five mainly components.

1) Electronic Health Record (EHR): is designed to represent data that accurately captures the state of the patient at all time. The EHR is composed of two main parts: Patient Profile Ontology (PPO) and Data Access Object (DAO) layer. The Patient Profile Ontology is crucial for our platform as it contents all relevant information about the patient, it include a range of information, including general information (age, sex, job, ethnic group, etc.), medical information (pathologies, allergies, treatments, surgical history, laboratory test results, etc.), medical measurements (blood pressure, weight, heart pulse, etc.) and lifestyle information (physical activity, eating habits, smoking, sleep, etc.). DAO Layer provides an abstract interface to the PPO. It is responsible of loading/adding data from/into the PPO.

2) Information Gathering System (IGS): it is designed to guide the clinicians in gathering relevant information process. The IGS provides a set of important questions for collecting cues about the state of the patient. These questions are dynamically adapted to the patient profile and take into account the answers given by the patient.

The IGS is composed of a Questionnaire Ontology (QO) and an Adaptive Engine (AE).

a) Questionnaire Ontology (QO): is designed to contain the questions which are used for gathering information. The construction of QO was inspired by the groundbreaking work Matt-Mouley Bouamrane presented in [13], [14]. It is

\footnotetext{
${ }^{2}$ American Heart Association

${ }^{3}$ European Society of Cardiology
}

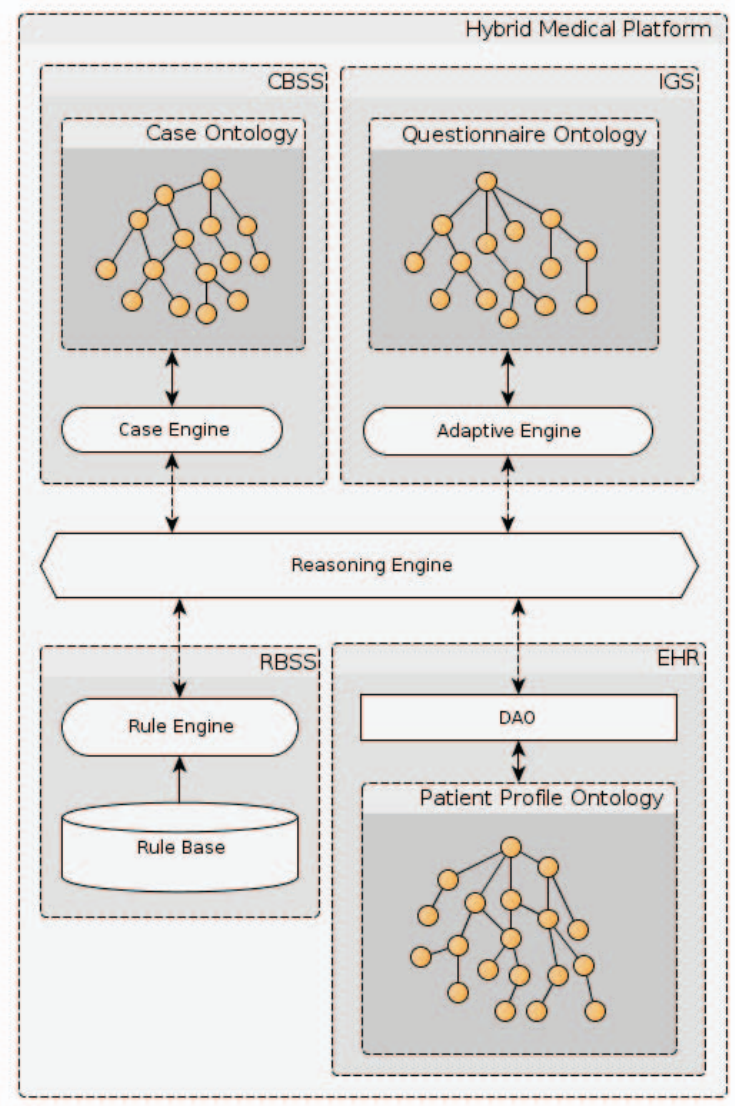

Fig. 1: Medical platform architecture

structured in four mainly concepts. Questionnaire, SubQuestionnaire, Question and PotentialAnswer. The QO is hierarchically structured of which the questions are structured under questionnaires and sub-questionnaires according to thematics (medical history, family history, current treatments, lifestyle information, etc.).

The concepts are inter-related through two types of properties namely: structural properties and adaptive properties.

(i) The structural properties: are used to describe the structure of the questionnaire. They include the following properties: hasParent, hasChild, hasSibling, hasExpectedAnswers, etc.

(ii) The adaptive properties: permit to determine the dynamic behaviour of the questionnaire. They include the following properties: ifTheAnswerIsEqualsTo, ifTheAnswerIsLessThan, ifTheAnswerIsGreaterThan, thenGoTo, etc. (We will give more details in the future paper). The questionnaires are created by domain experts (clinicians) through a user interface based on a clinical guidelines.

b) Adaptive Engine $(A E)$ : permits to adapt the questionnaire as per the patients profile. AE prompts only questions which are significant to the patient (e.g. AE doesn't prompt questions about the smoking habits if it is already mentioned in the patient profile that the patient is non-smoker). AE adapts the questions according to the formerly answers given per the patient using the adaptive properties. 
The gathered data is used by the reasoning engine for the decision making.

3) Rule-Based Decision Support System: is designed to apply the RBR. It composed of a rule base and an inference engine. The rule base is maintained by domain experts with the help of knowledge engineers who can add and update the clinical rules. These rules are described with Semantic Web Rule Language (SWRL).

4) Case-based Decision Support System: is designed to apply the CBR. It is composed of a case base and an reasoning engine. The case base is represented by an ontology (CaseOntology) which contents all cases treated in the past. In our approach, the case is consisted of three parts as follows:

a) Problem descriptor: is considered as an capture of patient profile at a specific time (i.e. temporal profile) since the patient profile evolves over time due to disease progression, lifestyle change, etc.

b) Solution: this part depend on the use of the platform. It could include diagnosis, treatments, protocols, etc.

c) Result: it could be the result obtained after applying the solution and/or the solution assessment or the clinician feedback.

5) Reasoning Engine: is the central component of the platform. It controls and coordinates the components of the platform (IGS, EHR, CBDSS, RBDSS) between them. Moreover, it acts as intermediary between the clinician and the rest of the components of the platform. It receives requests form the clinician, decides which component should be called to accomplish the task (e.g. IGS to gather missing information, CBDSS to retrieve similar cases, etc.) and then, it presents the results (e.g. similar cases, inferred conclusions, etc.) to the clinician at each step of the clinical process.

For more semantic and best knowledge sharing, the platform employs two additional ontologies: Guideline-Ontology (GO) and Domain-Ontology.

Guideline-Ontology $(G O)$ is used to model the knowledge related to the medical domain (cardiovascular disease diagnosis, diabetes diseases diagnosis, etc.). This knowledge are obtained from the textual medical guidelines and used for the creation of questionnaires and defining clinical rules.

Domain-Ontology (DO) is used to model the medical knowledge (disease, signs, treatments and relationships between concepts). DO provides a controlled vocabulary for ensuring integrability between different components of the platform and for best sharing knowledge with external platforms.

\section{B. The general functioning}

Fig. 2 illustrates the general functioning of the proposed platform.

The proposed platform could be used for assisting clinicians to diagnose complex medical cases.

Firstly, the IGS is used to help the clinician to gather relevant information about patient such as symptoms, medical

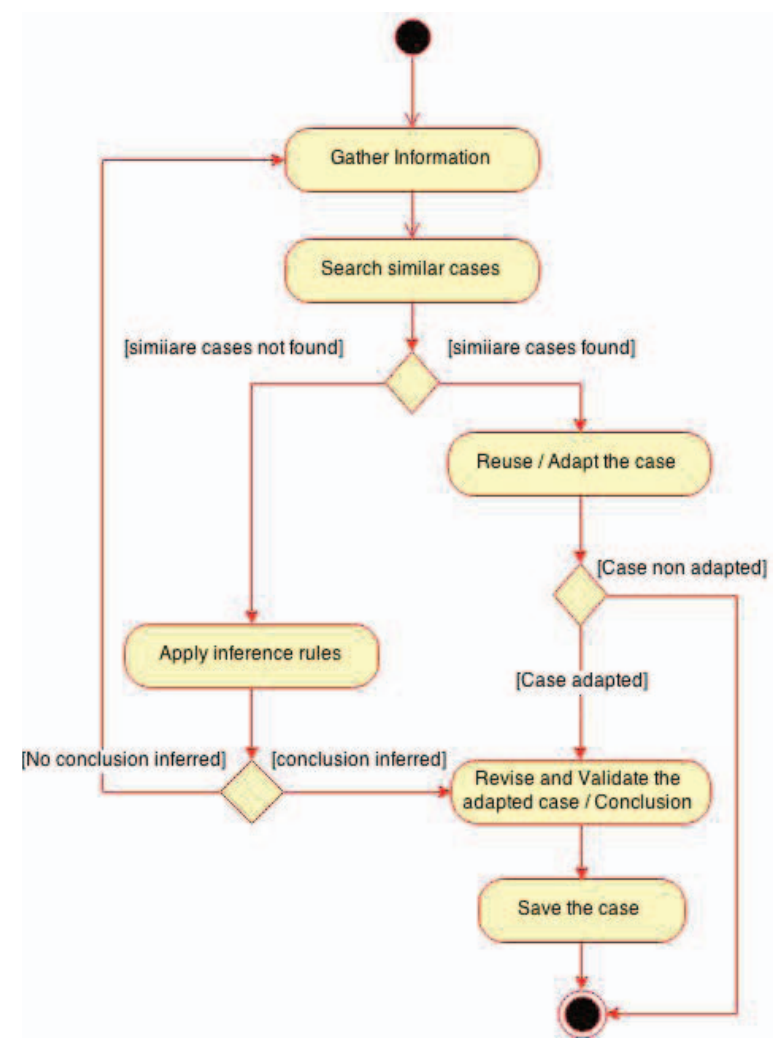

Fig. 2: The general functioning of the medical platform

history, family history, lifestyle information, etc. This is important, since the medical reasoning is based on the gathered information. The IGS generates an adaptive questionnaire which will guide the clinician in his medical reasoning. The questionnaire generated contains a fixed and extensive set of questions which are adapted to the patient profile and take into account the formerly answers given by the patient. This way ensure that no relevant question is forgotten to be asked.

Noting that for each question posed, the information gathered is used by the reasoning engine to seek for the corresponding diagnosis either by applying the CBR or the RBR.

The reasoning engine evokes first the CBDSS, based on the CBR cycle as proposed by A. Aamodt and E. Plaza in [18]. In the first step, the CBDSS matches the patient profile that contains the information collected with the sources cases stored in the case base in order to retrieve similar cases. If similar cases are found, the most relevant case is proposed to the clinician of which the information and knowledge contained in this latter may be reused to solve the actual clinical case. This step might possibly entail an adaptation step of the solution of the proposed case, considering the differences between both problematic situations.

If so, the solution of the adapted case is revised and validated either by the same clinician or per another one (e.g. clinician with more experience).

Once the case (problem, confirmed solution and results) is validate. The new case is stored in the case base in the hope of being useful to treat new clinical cases. 
Otherwise (i.e. no similar case has been found) the reasoning engine invokes the RBDSS. This, processes the rule base in order to infer a solution. If the RBDSS doesn't find suitable rules to apply by lack of information, the reasoning engine orders to the IGS to gather more information in order to exploit new ways of reflection and this cycle is repeated until a best solution is reached.

\section{CONCLUSION}

In this paper, we have presented our approach for designing a generic medical platform. The proposed platform can play a major role in assisting clinicians in their clinical reasoning including gathering information and decision making.

The main specificity of our platform, is the combining of two modes of reasoning (CBR and RBR) in a cyclic process with gathering relevant information in each cycle for the process. In which the best out of CBR and RBR is exploited to enhance the decision making accuracy.

Furthermore, the use of the platform within a medical community can be beneficial since each individual (clinician) of the community can exploit his own experience or others' experiences. Thus, the medical community can benefit from the experience of each clinician and vice versa.

\section{REFERENCES}

[1] K. Hoffman, "A comparison of decision-making by expert and novice nurses in the clinical setting, monitoring patient haemodynamic status post abdominal aortic aneurysm surgery," Ph.D. dissertation, University of Technology, Sydney, Australia, 2007.

[2] M. Kraischsk and M. Anthony, "Benefits and outcomes of staff nurses' participation in decision making," Journal of Nursing Administration, vol. 31, no.1, pp. 16-23, 2001.

[3] S. Lauri, S. Salantera, K. Chalmers, S. Ekman, H. Kim, S. Hesook, S. Kapelli and M. MacLeod, "An exploratory study of clinical decisionmaking in five countries," Journal of Nursing Scholarship, vol. 33, no. 1, pp. 83-90, 2001.

[4] M. J. Goolsby, L. Grubbs, "Advanced Assessment: Interpreting Findings and Formulating Differential Diagnoses", 2nd Ed., F.A. Davis Company, 2011.

[5] E. H. Shortliffe and J. J. Cimino, Biomedical informatics: computer applications in health care and biomedicine, Medical Informatics, Springer-Verlag, New York, 2001.

[6] E. S. Berner and T. J. La Lande, Overview of Clinical Decision Support Systems, Chapter 1 of Clinical Decison Support Systems theory and Practice, 2nd Ed., Health informatics series, pp. 3-22, 2007.

[7] A. Wright, D. W. Bates, B. Middleton, T. Hongsermeier, V. Kashyap, S. M. Thomas, D. F. Sittig, "Creating and sharing clinical decision support content with Web 2.0: Issues and examples," Journal of Biomedical Informatics, vol. 42, no. 2, pp. 334-346, 2009.

[8] D. Dinevski, U. Bele, T. Sarenac, U. Rajkovic and O. Sustersic, Clinical Decision Support Systems, Chapter 8, Telemedicine Techniques and Applications book edited by Georgi Graschew and Stefan Rakowsky, ISBN 978-953-307-354-5, June 20, 2011.

[9] F. Le Ber, J. Lieber and A. Napoli, Les systmes á base de connaissances Encyclopdie de l'informatique et des systèmes d'information, J. Akoka and Comyn-Wattiau (Ed.), pp. 1197-1208, 2006.

[10] M.K. Goldstein, R.W. Coleman, S.W. Tu, R.D. Shankar, M.J. O'Connor, M.A. Musen, S.B. Martins, P.W. Lavori, M.G. Shlipak, E. Oddone, AA. Advani, P. Gholami, BB. Hoffman Translating research into practice: organizational issues in implementing automated decision support for hypertension in three medical centers J Am Med Inform Assoc 11(5): pp. 368-376, 2004.
[11] S. Lai, M.K. Goldstein, et al., Insights from Testing the Accuracy of Recommendations from an Automated Decision Support System for Primary Hypertension: ATHENA DSS MEDINFO CD: 1706, 2004.

[12] J.H.. Carter, Design and Implementation Issues Chapter 4 Clinical Decision Support Systems theory and Practice Second Edition HEALTH INFORMATICS SERIES, 2007.

[13] M.M. Bouamrane, A. Rector, M. Hurrell, Ontology-Driven Adaptive Medical Information Collection System 17th International Symposium, ISMIS 2008 Toronto, Canada, May 20-23, 2008.

[14] M.M. Bouamrane, A. Rector, M. Hurrell, Gathering Precise Patient Medical History with an Ontology-driven Adaptive Questionnaire Computer-Based Medical Systems, 2008. CBMS '08. 21st IEEE International Symposium on 17-19 June 2008.

[15] Z. El Balaa and T. Ralph, Case-based decision support and experience management for ultrasonography, In German Workshop on Experience Management. GWEM03, 2003

[16] Z. El Balaa, A. Strauss, P. Uziel, K. Maximini and R. Traphner, "FM-Ultranet: a Decision Support System using Case-Based Reasoning, Applied to Ultrasonography", Workshop on Case-Based Reasoning in the Health Sciences, Trondheim, Norway, June 2003.

[17] B. Chakraborty, S.I. Srinivas, P. Sood, V. Nabhi and D. Ghosh CaseBased reasoning methodology for diagnosis of swine flu 2011 IEEE GCC Conference and Exhibition (GCC), Dubai, United Arab Emirates, February 19-22, 2011.

[18] A. Aamodt, and E. Plaza, Case-based reasoning: Foundational issues, methodological variations, and system approaches AI Communications 7, pages 39-59, 1994

[19] S. Begum A case-based reasoning system for the diagnostic of individual sensitivity to stress in psychophysiology Mlardalen University Press Licentiate Theses, 2009.

[20] H.T. Wang and A. Uz Tansel, MedCase: A Template Medical Case Store for Case-Based Reasoning in Medical Decision Support IEEE/ACM International Conference on Advances in Social Networks Analysis and Mining. 2013

[21] A. Cordier, B. Mascret and A. Mille, Étendre les possibilits du raisonnement à partir de cas grâce aux traces 7ème atelier de Raisonnement á Partir de Cas, 2009.

[22] A. Rector and J. Rogers, Ontological and Practical Issues in Using a Description Logic to Represent Medical Concept Systems: Experience from GALEN Reasoning Web, Lecture Notes in Computer Science, 4126/2006:197-231, 2006.

[23] A.B. Eshete, Integrated Case-Based and Rule-Based Reasoning for Decision Support M.Sc., Information Systems, Norwegian University of Science and Technology, 2009.

[24] P.C. Sherimon, P.V. Vinu, R. Krishnan and Y. Takroni, Ontology Based System Architecture to Predict the Risk of Hypertension in Related Diseases IJIPM: International Journal of Information Processing and Management, Vol. 4, No. 4, pages 44-50, 2013.

[25] P. C. Sherimon, P.V. Vinu, R. Krishnan, Y. Takroni, Y. AlKaabi, Y. AlFars, Adaptive Questionnaire Ontology in Gathering Patient Medical History in Diabetes Domain Proceedings of the First International Conference on Advanced Data and Information Engineering (DaEng2013) Lecture Notes in Electrical Engineering Volume 285, pages 453460, 2014

[26] K. Farooq, A. Hussain, S. Leslie, C. Eckl, and W. Slack, ”Ontology Driven Cardiovascular Decision Support System", In: 5th International Conference on Pervasive Computing Technologies for Healthcare (PervasiveHealth), pp.283-286, 2011.

[27] N. F. Noy, D. L. McGuinness, "Ontology Development 101: A Guide to Creating Your First Ontology" Stanford University, 2005.

[28] S. Begum, M. U. Ahmed, P. Funk and M. Folke, "Case-Based Reasoning Systems in the Health Sciences A Survey of Recent Trends and Developments", IEEE Transactions on Systems, Man, and Cybernetics, Part C 7 (1) (2010) 39-59. 